\title{
КЛАССИФИКАЦИЯ ОДНО- И ДВУМЕРНЫХ СИГНАЛОВ МЕТОДАМИ НОРМАЛИЗАЦИИ И НОРМАЛЬНОГО ПРЕОБРАЗОВАНИЯ
}

\author{
РЫБИН А. И., МЕЛЬНИК А. Д., НИЖЕБЕЦКАЯ Ю. Х., СУШКО И. А., ЛИТВИНЦЕВ С. Н. \\ Национальный технический университет Украины \\ «Киевский политехнический институт», \\ Украина, Киев, 03056, пр-т Победы 37
}

\begin{abstract}
Аннотация. Предложены различные способы классификации одномерных сигналов при помощи методов нормализации, включающих в себя нормализацию по уровню и по шагу, и нормального преобразования. Рассмотрено применение нормализации по уровню и нормального преобразования для двумерных сигналов. Приведены алгоритмы формирования фильтра и классификации для рассмотренных вариантов. Описан алгоритм прямого формирования матричного оператора нормального преобразования для одномерного сигнала. Применение рассмотренных способов проиллюстрировано на примерах. Учтена возможность изменения масштаба входных сигналов
\end{abstract}

Ключевые слова: ортогональное преобразование; согласованная фильтрация; классификация; нормализация; нормальное преобразование; коэффициент трансформант

\section{1. ВВЕДЕНИЕ}

Решение задачи распознавания (классификации) сигналов и образов приобретает все большее значение. Методы решения этой задачи разнообразны $[1,2]$. Часто решение задачи классификации сигналов выполняется с использованием согласованной фильтрации [3] или ортогонального преобразования $[4,5]$.

Классическая согласованная фильтрация не дает численных оценок подобия между исследуемым и эталонным сигналами, т.к. она только регистрирует наличие или отсутствие эталонного сигнала на фоне белого шума. Ортогональные преобразования (ОП) позволяют получить такие численные оценки для случаев, когда одна из базовых функций ОП совпадает по форме с эталонным сигналом. Например, коэффициент гармоник, вычисляемый по спектру Фурье для исследуемого сигнала «похожего» на гармонику. Таким образом, распознава- ние с численной оценкой подобия зависит от наличия нормального преобразования [4].

Для решения задачи распознавания необходимо найти спектр исследуемого сигнала с использованием нормального ОП. Сравнение спектров исследуемого и эталонного сигналов дает численную оценку их подобия.

Процедуру численной оценки различия между эталоном и исследуемым сигналами возможно интерпретировать как согласованную многоканальную фильтрацию [4], количество каналов фильтра которой равно порядку матричного оператора дискретного ортогонального преобразования (ДОП). Таким образом, для классификации на базе такой фильтрации, являющейся вариантом специальной согласованной фильтрации, необходимы способы построения ОП, в которых одна из базовых функций совпадает с эталонным сигналом. Задача имеет несколько вариантов решения: 